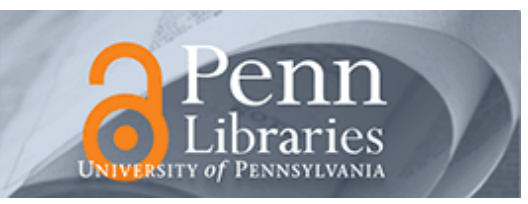

University of Pennsylvania

ScholarlyCommons

\title{
Simultaneous Estimation and Segmentation of T1 Map for Breast Parenchyma Measurement
}

\author{
Ye Xing \\ University of Pennsylvania, yexing@seas.upenn.edu \\ Yangming $\mathrm{Ou}$ \\ University of Pennsylvania, ouya@seas.upenn.edu \\ Sarah Englander \\ University of Pennsylvania, sarah.englander@uphs.upenn.edu \\ Mitchell D. Schnall \\ University of Pennsylvania, Mitchell.Schnall@uphs.upenn.edu \\ Dinggang Shen \\ University of Pennsylvania, Dinggang.Shen@uphs.upenn.edu
}

Follow this and additional works at: https://repository.upenn.edu/be_papers

Part of the Bioimaging and Biomedical Optics Commons

\section{Recommended Citation}

Xing, Y., Ou, Y., Englander, S., Schnall, M. D., \& Shen, D. (2007). Simultaneous Estimation and Segmentation of T1 Map for Breast Parenchyma Measurement. Retrieved from https://repository.upenn.edu/be_papers/ 139

Ye Xing, Yangming Ou, Sarah Englander, Mitchell D. Schnall, Dinggang Shen: "Simultaneous Estimation and Segmentation of T1 Map for Breast Parenchyma Measurement". IEEE International Symposium on Biomedical Imaging (ISBI) 2007: pp. 332-335

http://ieeexplore.ieee.org/xpls/abs_all.jsp?tp=\&arnumber=4193290\&isnumber=4193197

This paper is posted at ScholarlyCommons. https://repository.upenn.edu/be_papers/139

For more information, please contact repository@pobox.upenn.edu. 


\title{
Simultaneous Estimation and Segmentation of T1 Map for Breast Parenchyma Measurement
}

\begin{abstract}
Breast density has been shown to be an independent risk factor for breast cancer. In order to segment breast parenchyma, which has been proposed as a biomarker of breast cancer risk, we present an integrated algorithm for simultaneous T1 map estimation and segmentation, using a series of magnetic resonance (MR) breast images. The advantage of using this algorithm is that the step of T1 map estimation (E-Step) and the step of T1 map based tissue segmentation (S-Step) can benefit each other. Since the estimated T1 map can be noisy due to the complexity of T1 estimation method, the tentative tissue segmentation results from S-Step can help perform the edge-preserving smoothing on the estimated T1 map in E-Step, thus removing noises and also preserving tissue boundaries. On the other hand, the improved estimation of T1 map from E-Step can help segment breast tissues in a more accurate and less noisy way. Therefore, by repeating these steps, we can simultaneously obtain better results for both T1 map estimation and segmentation. Experimental results show the effectiveness of the proposed algorithm in breast tissue segmentation and parenchyma volume measurement.
\end{abstract}

\section{Keywords}

Breast cancer, parenchyma, T1 map, image segmentation, estimation

\section{Disciplines}

Bioimaging and Biomedical Optics

\section{Comments}

Ye Xing, Yangming Ou, Sarah Englander, Mitchell D. Schnall, Dinggang Shen: "Simultaneous Estimation and Segmentation of T1 Map for Breast Parenchyma Measurement". IEEE International Symposium on Biomedical Imaging (ISBI) 2007: pp. 332-335

http://ieeexplore.ieee.org/xpls/abs_all.jsp?tp=\&arnumber=4193290\&isnumber=4193197 


\title{
SIMULTANEOUS ESTIMATION AND SEGMENTATION OF $T_{1}$ MAP FOR BREAST PARENCHYMA MEASUREMENT
}

\author{
Ye Xing ${ }^{1}$, Yangming $\mathrm{Ou}^{1}$, Sarah Englander ${ }^{2}$, Mitchell Schnall ${ }^{2}$, Dinggang Shen ${ }^{2}$ \\ ${ }^{1}$ Department of Bioengineering, ${ }^{2}$ Department of Radiology, University of Pennsylvania
}

\begin{abstract}
Breast density has been shown to be an independent risk factor for breast cancer. In order to segment breast parenchyma, which has been proposed as a biomarker of breast cancer risk, we present an integrated algorithm for simultaneous $T_{1}$ map estimation and segmentation, using a series of magnetic resonance (MR) breast images. The advantage of using this algorithm is that the step of $T_{1}$ map estimation (E-Step) and the step of $T_{1}$ map based tissue segmentation (S-Step) can benefit each other. Since the estimated $T_{1}$ map can be noisy due to the complexity of $T_{1}$ estimation method, the tentative tissue segmentation results from S-Step can help perform the edge-preserving smoothing on the estimated $T_{1}$ map in E-Step, thus removing noises and also preserving tissue boundaries. On the other hand, the improved estimation of $T_{1}$ map from E-Step can help segment breast tissues in a more accurate and less noisy way. Therefore, by repeating these steps, we can simultaneously obtain better results for both $T_{1}$ map estimation and segmentation. Experimental results show the effectiveness of the proposed algorithm in breast tissue segmentation and parenchyma volume measurement.
\end{abstract}

Index Terms - Breast cancer, parenchyma, $T_{1}$ map, image segmentation, estimation

\section{INTRODUCTION}

Breast cancer is the most common cancer in women. Parenchyma tissue and fat tissue are the two major components of the breast, and extensive research on breast cancer has shown that the woman with a dense breast (or high parenchyma volume) is in a high risk of developing breast cancer $[1,2]$. Quantitative analysis of parenchyma tissue by MR imaging could assess the parenchyma volume, thus potentially assisting the diagnosis of breast cancer in early stages. Moreover, it can quantitatively monitor how drugs or low-fat diets take effects on changing the volume of parenchyma tissue, thus helping doctors specify a treatment for effectively lowering the risk of developing breast cancer. Accordingly, it is important to accurately segment parenchyma from breast.

Breast MR imaging can provide three-dimensional images with high soft tissue contrast, thus enabling the segmentation and quantitative measurement of parenchyma. So far, there are mainly two types of methods proposed for segmenting parenchyma tissue from breast MR images, i.e., intensity-based segmentation and tissue property based segmentation.

Intensity-based segmentation assumes that different breast tissues have different intensities in the breast MR images. Although this type of segmentation methods is easy to implement and thus widely used, it has its own drawbacks since the same type of tissue may have variant intensities in an image mainly due to image inhomogeneity. Accordingly, the finally measured parenchyma volume might be not sufficiently accurate for guiding cancer diagnosis and treatment.

Tissue property based segmentation uses MR physics to characterize the intrinsic properties of tissues, i.e., using $T_{1}$ value to differentiate parenchyma from fat, since it is believed that different tissues have different $T_{1}$ values [3]. Accordingly, Brady et al [4] developed a breast tissue segmentation method based on the $T_{1}$ values estimated using the fast spoiled gradient echo pulse sequence. This kind of $T_{1}$ measurement techniques based on multiple excitation flip angles are sensitive to the applied flip angles, and can vary throughout the imaging volume [5]. We use an inversion recovery imaging technique to acquire data, which is less sensitive to $B_{1}$ homogeneity. Notice that, for both methods described above, the accurate estimation of $T_{1}$ values is extremely important, since it is the basis for successful segmentation of parenchyma tissue.

In this study, we propose a novel method which integrates the estimation and the segmentation of $T_{1}$ values into a single framework, thus allowing them to benefit each other. In particular, the tentative segmentation results can be used to constrain the estimation of $T_{1}$ values, by requiring the same tissue (in the neighborhood) to have spatially smooth $T_{1}$ values, which can effectively improve the estimation of $T_{1}$ values by reducing noises. In the meanwhile, the improved estimation of $T_{1}$ values can help segment breast tissue in a more accurate and less noisy way. Therefore, by iteratively performing these steps, we can simultaneously obtain better results for $T_{1}$ value estimation and also tissue segmentation. This will greatly help the measurement of parenchyma tissue, and eventually aid the diagnosis and treatment of breast cancer. 


\section{METHOD}

Before detailing our simultaneous $T_{1}$ map estimation and segmentation algorithm, the acquisition of MR breast images for measurement of parenchyma volume will be first introduced. Also, the method for estimating voxel-wise $T_{1}$ value, based on acquired image series of each breast, will be provided.

\subsection{Data acquisition}

The image data were acquired using a 3D inversion recovery spoiled gradient echo sequence [6]. For each breast, five series of $3 \mathrm{D}$ images, $\left\{f^{m}, m=1, \ldots, 5\right\}$, were acquired by using five different sets of inversion time $T_{\mathrm{i}}$ and repetition time $T_{\mathbf{R}}$, i.e., $\left\{\left(T_{\mathbf{i}}^{m}, T_{\mathbf{R}}{ }^{m}\right), m=1, \ldots, 5\right\}=\{(1600,1880),(800$, $1080),(400,680),(200,480),(140,420)\}$ in a unit of $m s$. The observation flip angle is fixed to $\alpha=20^{\circ}$ during all data acquisition.

The intensities in each acquired image $f^{m}$ can also be theoretically represented by a solution of Bloch equation as defined next. Given $T_{\mathbf{i}}{ }^{m}, T_{\mathbf{R}}{ }^{m}$ and $\alpha$ for each image $f^{m}$, the theoretical intensity at each voxel $v$ is $\hat{f}^{m}(v)$, which is a function of three position-dependent parameters, $T_{1}$ value $T_{1}(v)$, equilibrium magnetization $S_{0}(v)$, and inversion pulse flip angle $\beta(v)$. Notice that $T_{1}(v)$ is tissue-dependent, while two parameters $S_{0}(v)$ and $\beta(v)$ are generally tissue-independent. The relationship among these parameters is mathematically defined as follows:

$$
\begin{aligned}
\left.\hat{f}^{m}(v)\right|_{T_{1}(v), S_{0}(v), \beta(v)} & =S_{0}(v)\left(1-\exp \left(-\frac{T_{\mathbf{i}}^{m}}{T_{1}(v)}\right)\right) \\
+ & \frac{S_{0}(v)\left(1-\exp \left(-\frac{T_{\mathbf{R}}^{m}}{T_{1}(v)}\right)\right) \exp \left(-\frac{T_{\mathbf{i}}^{m}}{T_{1}(v)}\right) \cos (\beta(v))}{1-\exp \left(-\frac{T_{\mathbf{R}}^{m}}{T_{1}(v)}\right) \cos (\alpha)}
\end{aligned}
$$

This equation indicates that three parameters $T_{1}(v), S_{0}(v)$, and $\beta(v)$ on each voxel $v$ can be estimated from five acquired intensities, $\left\{f^{m}(v), m=1, \ldots, 5\right\}$, by minimizing the fitting errors as defined in Subsection 2.2.1.

\subsection{Simultaneous $T_{1}$ map estimation and segmentation}

Although it seems straightforward to estimate $T_{1}$ map, i.e., estimating $T_{1}$ value independently for each voxel in the image space, however, the estimated $T_{1}$ map can be spatially very noisy, due to the very complex equations given in (1). Thus, it is important to constrain the spatial smoothness of the estimated $T_{1}$ map during the estimation procedure. However, if using a simple isotropic smoothing filter, i.e., Gaussian filter, the boundary between different breast tissues can be blurred. Therefore, it is important to know the tissue types when smoothing the estimated $T_{1}$ map, in order to smooth only the voxels with same tissue class.
We propose a novel integrated framework that simultaneously estimates and segments the $T_{1}$ map. The segmentation of $T_{1}$ map helps perform the edge-preserving smoothing on the tentatively estimated $T_{1}$ map, while the improved $T_{1}$ map estimation helps produce a more accurate and less noisy tissue segmentation. By iteratively repeating these steps, we can achieve both better estimation and segmentation of $T_{1}$ map. The overview of our proposed algorithm is summarized next, and details for each important step will be provided in the subsequent subsections.

\section{Summary of algorithm:}

(1) Use random values as initialization for $T_{1}(v), S_{0}(v)$ and $\beta(v)$, and then independently estimate their true values by minimizing the errors between the practically acquired intensities $f^{m}(v)$ and theoretically estimated intensities $\hat{f}^{m}(v)$ as detailed in Subsection 2.2.1.

(2) S-Step: Segment the currently estimated $T_{1}$ map into two classes, i.e., parenchyma and fat, by using a tissue segmentation algorithm such as fuzzy segmentation algorithm described in Subsection 2.2.2.

(3) E-Step: (a) Perform an edge-preserving smoothing on the currently estimated $T_{1}$ map, therefore achieving a smoothed version of $T_{1}$ map, as detailed in Subsection 2.2.3. Notice that the parameters $S_{0}(v)$ and $\beta(v)$ will be also made spatially smooth by using an isotropic Gaussian filter, since they are likely tissue-independent. (b) Use the smoothed $T_{1}$ map as an initialization to obtain a new estimation for $T_{1}$ map, by similarly minimizing the fitting error as used in Step (1).

(4) In E-Step, if the fitting error reaches a predefined value, stop; otherwise, go to S-Step and E-Step again for the next iteration of tissue segmentation and $T_{1}$ map estimation.

\subsection{1. $T_{1}$ map estimation}

The estimation of $T_{1}$ map is formulated as an energy minimization problem. Basically, we required the theoretically estimated intensities $\hat{f}^{m}(v)$ should best fit with the practically acquired intensities $f^{m}(v)$ on each voxel $v$. Mathematically, it can be defined as follows:

$$
E=\sum_{v} \sum_{m=1}^{5}\left(f^{m}(v)-\left.\hat{f}^{m}(v)\right|_{T_{1}(v), S_{0}(v), \beta(v)}\right)^{2}
$$

Note that $E$ is a function of $T_{1}(v), S_{0}(v)$, and $\beta(v)$, the objective function $E$ can be minimized once the optimal set of $T_{1}(v), S_{0}(v)$, and $\beta(v)$ is found.

A gradient-based optimization algorithm is used in this study to search for suboptimal solutions, based on the initializations provided for $T_{1}(v), S_{0}(v)$, and $\beta(v)$. As indicated above, random values will be provided in the first iteration of $T_{1}$ map estimation, while in other iterations the previously estimated $T_{1}$ map after edge-preserving smoothing will be used as an initialization for new $T_{1}$ map estimation. 


\subsubsection{Fuzzy tissue segmentation}

Segmentation of breast into parenchyma and fat is our final goal of measuring the volume of parenchyma, for helping diagnosis and treatment of breast cancer. Besides, the tentative segmentation results produced during the $T_{1}$ map estimation procedure can also allow us to perform an edge-preserving smoothing for the iteratively estimated $T_{1}$ map. Therefore, it is important to develop a reasonable method for tissue segmentation.

Accordingly, Fuzzy C-Means (FCM) is used to segment breast tissues, based on the currently estimated $T_{1}$ map. The advantage of using this algorithm is that it could provide a continuous membership value ranging from 0 (i.e., pure fat) to 1 (i.e., pure parenchyma) for each voxel, which is important for dealing with the partial volume effect in the breast MR images. Notice that the partial volume effect is common in breast MR imaging, and it is resulted due to the contribution of both fat and parenchyma into the intensity of the same voxel. Thus, by using FCM, the estimated memberships of fat and parenchyma in a voxel directly represent the percentages of fat and parenchyma within this voxel. Importantly, this kind of advantage cannot be easily achieved by simply using a traditional global histogram based segmentation algorithm [3].

\subsubsection{Edge-preserving smoothing}

The energy function $E$ in Eq. (2) is very complex, thus the estimated $T_{1}$ map can be very noisy due to the lack of spatial smoothness constraints on estimated $T_{1}$ map. As mentioned before, if using an isotropic smoothing filter, it can blur boundaries between different breast tissues. To avoid this, we propose to use an edge-preserving smoothing filter, which would smooth $T_{1}$ values only for the voxels with the same tissue class. Notice that the tissue class for each voxel is determined by tissue segmentation results using FCM.

In this study, an edge-preserving Gaussian filter is applied to the $T_{1}$ map. This edge preserving Gaussian filter will only smooth the pixels which belong to the same class according to the FCM-based tissue segmentation step, while the pixels with different class will not get the chance to be smoothed together. This step solves the problem of over-smoothing between different types of tissues, thus giving more accurate estimation of $T_{1}$ map. Notice that, with the $T_{1}$ map smoothed, it can be used as a good initialization for next iterative estimation of $T_{1}$ map.

\section{RESULTS}

Two sets of experiments are provided for demonstrating the performance of the proposed algorithm in simultaneous estimation and segmentation of $T_{1}$ map. Firstly, the average local variance of parenchyma $T_{1}$ values is provided to show the effectiveness of our simultaneous $T_{1}$ map estimation and segmentation framework. Secondly, the comparison between algorithm-based segmentation and manual segmentation is provided for validating the accuracy of proposed tissue segmentation algorithm.

\subsection{Local variance of parenchyma $T_{1}$ values}

The local variance of parenchyma $T_{1}$ values measures the variance of $T_{1}$ values within a neighborhood around a specific parenchyma voxel. The iterative decreases of local variance in our joint estimation and segmentation framework represent the smoothness as well as relative accuracy of $T_{1}$ map estimated.

As shown in Fig 1, the local variance generally decreases with the increase of iterations, and becomes relatively stable after a sufficient number of iterations. The results for two patients are particularly provided. For comparison, variances are normalized by the corresponding initial variances. This experiment indirectly shows the accuracy of $T_{1}$ map estimation and the importance of using iterative optimization to improve the estimation.

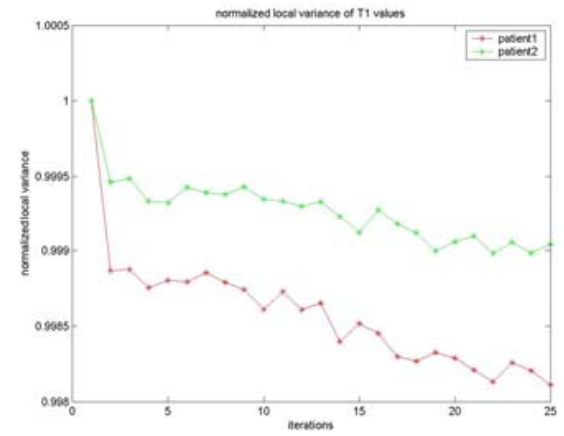

Fig. 1. Iterative decreases of normalized local variance of parenchyma $T_{1}$ values during the $T_{1}$ map estimation and segmentation procedure, for two particular patients.

\subsection{Validation}

To validate the accuracy of the proposed tissue segmentation algorithm, the algorithm-based segmentation results are compared with the manual segmentation results by two human raters. Both overlay percentages and volume errors are provided in Table 1.

For visual inspection of tissue segmentation by the proposed method, the five series of breast images along with the result produced by our automatic segmentation method are provided in Fig 2. Two manual segmentation results and our automatic segmentation result are shown in Fig 3.

From Table 1, we could see that overlay percentage between automatic segmentation and manual segmentation is generally around $90 \%$, while volume error is generally around $10 \%$. The differences between the automatic segmentations and manual segmentations are statistically similar to those of two human raters. That means, our $T_{1}$ map based automatic segmentation has a great potential of segmenting parenchyma tissue as good as human raters. 

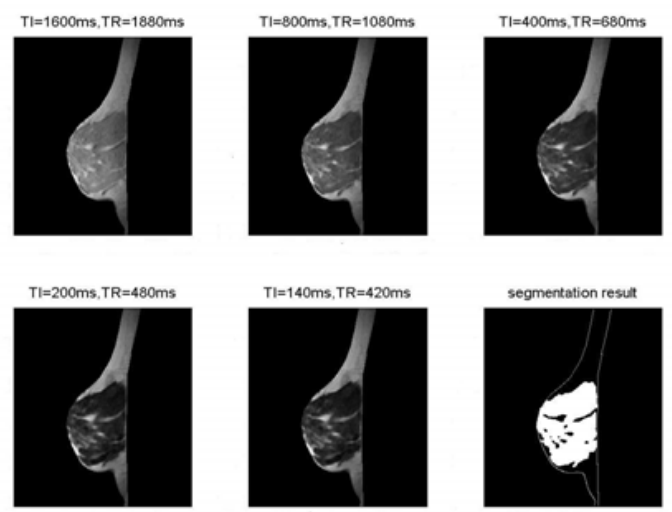

Fig. 2. Original images and our segmentation result (bottom right one). White regions in the bottom right image represent the segmented parenchyma in the breast.
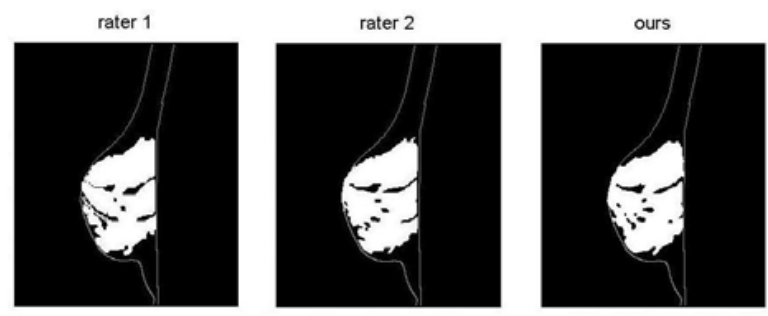

Fig. 3. The respective segmentation results by rater 1 , rater 2 , and our segmentation algorithm (from left to right). The white regions in each image are the segmented parenchyma tissue in the breast.

Table 1. Overlay percentages and volume errors between algorithm-based segmentations and manual segmentations. $\mathrm{R} 1$ denotes the human rater 1, and R2 denotes the human rater 2 .

\begin{tabular}{|l|c|c|c|}
\hline & Our vs. R1 & Our vs. R2 & R1 vs. R2 \\
\hline Overlay & $88.0 \%$ & $91.8 \%$ & $89.2 \%$ \\
\hline Volume error & $12.8 \%$ & $8.6 \%$ & $11.5 \%$ \\
\hline
\end{tabular}

\section{DISCUSSION}

We have proposed an integrated framework for simultaneous estimation and segmentation of $T_{1}$ map, using a series of MR breast images acquired with different inversion time and repetition time from the same subject. In this framework, the E-Step of $T_{1}$ map estimation and the S-Step of $T_{1}$ map based segmentation benefit each other, thus simultaneously achieving better results for both $T_{1}$ map estimation and segmentation. In particular, the tentative segmentation result from S-Step can help perform the edge-preserving smoothing on the estimated $T_{1}$ map, thus removing noises as well as preserving tissue boundaries.
Meanwhile, the improved estimation of $T_{1}$ map can help segment breast tissue in a more accurate and less noisy way. By repeating these steps, better results for $T_{1}$ map estimation and tissue segmentation can be simultaneously achieved. As demonstrated by experimental results, our automatic segmentation based on $T_{1}$ map has potential as a computer calculated biomarker of breast cancer risk.

In the future, more validations, i.e., using phantom data with ground-truth breast tissue volumes, will be performed, therefore potentially avoiding any biases or errors produced by human raters when validating our tissue segmentation algorithm. Also, more breast image datasets will be used to test the performance of the proposed $T_{1}$ map estimation and segmentation algorithm.

\section{REFERENCES}

[1] J. Brisson, F. Merletti, N. L. Sadowsky, J. A. Twaddle, A. S. Morrison, and P. Cole, "Mammographic features of the breast and breast cancer risk," American Journal of Epidemiology, vol. 115, pp. 428-437, 1982.

[2] J. W. Byng, M. J. Yaffe, R. A. Jong, R. S. Shumak, G. A. Lockwood, D. L. Tritchler, and N. F. Boyd, "Analysis of mammographic density and breast cancer risk from digitized mammograms," Radiographics, vol. 18, pp. 1587-1598, 1998.

[3] R. C. Boston, M. D. Schnall, S. A. Englander, J. R. Landis, and P. J. Moate, "Estimation of the content of fat and parenchyma in breast tissue using MRI T1 histograms and phantoms," Magnetic Resonance Imaging, vol. 23, pp. 591-599, 2005.

[4] G. Ketsetzis and M. Brady, "Automatic segmentation of T1 parametric maps of breast MR images via a hidden Markov random field," presented at Proceedings of Medical Image Understanding and Analysis, 2004.

[5] G. Ketsetzis and M. Brady, "Improved T1 estimation in contrast enhanced MRI for pathology detection using optimum flip angle acquisitions," presented at Proceedings of Medical Image Understanding and Analysis (MIUA), 2004.

[6] T. K. F. Foo, A. M. Sawyer, W. H. Faulkner, and D. G. Mills, "Inversion in the Steady State: Contrast Optimization and Reduced Imaging Time with Fast Three-dimensional Inversion-Recovery-prepared GRE Pulse Sequences," Radiology, vol. 191, pp. 85-90, 1994. 